\title{
CAPÍTULO II
}

\section{PROCESO DE DIRECCIÓN ESTRATÉGICO Y RESPONSABILIDAD SOCIAL EMPRESARIAL PARA POTENCIAR EL TURISMO EN TIEMPOS EMERGENTES}

\author{
Osmany Pérez Barral \\ Doctor en Ciencias Económicas, docente de la Pontificia Universidad Católica \\ del Ecuador Sede Ambato; ORCID: 0000-0002-4735-483X. Correo electrónico: \\ operez@pucesa.edu.ec.
}

\section{Mónica del Rocío Alarcón Quinapanta}

Doctora en Ciencias Técnicas; Universidad Regional Autónoma de Los Andes; ORCID: 0000-0003-2510-4773. Correo electrónico: monyalarcon7@gmail. com.

\section{Jorge Vladimir Núñez Grijalva}

Doctor en Ciencias Jurídicas; Pontificia Universidad Católica del Ecuador Sede Ambato; ORCID: 0000-0001-5335-1160. Correo electrónico: vhernandez@ pucesa.edu.ec.

\section{Varna Hernández Junco \\ Doctora en Ciencias Técnicas; Pontificia Universidad Católica del Ecuador Sede Ambato; ORCID: 0000-0001-7864-6723. Correo electrónico: vhernandez@ pucesa.edu.ec.}

\section{Resumen}

El turismo es uno de los sectores de la economía que ha sido más golpeado en tiempos de pandemia, su repercusión ha sido de un impacto muy hostil y directo desde el punto de vista económico y de Responsabilidad Social Empresarial para con los trabajadores del sector y los clientes. En este sentido, la investigación tuvo como objetivo el diseño de un Proceso de Dirección Estratégico para el desarrollo y reactivación del sector del turismo en tiempos emergentes con responsabilidad social, con la finalidad, de que las organizaciones estén preparadas con tiempo para responder a las necesidades de los clientes y la sociedad en función de sus economías, finanzas y responsabilidad social, pues en muy improbable que los gerentes y directivos puedan estimar la duración de este 
tipo de fenómeno social. Para lo cual, la investigación realizada es de tipo descriptivaexplicativa con un enfoque mixto, lo cual permitió, transformar datos en información oportuna para tomar decisiones. Asimismo, se emplearon los métodos análisis y síntesis para el desarrollo del fundamento teórico y metodológico del estudio, dejándose explícito a partir de ello, la propuesta estratégica para el desarrollo del turismo en un contexto específico de emergencia, a partir de una proyección que está enfocada en la gestión y servicio al cliente interno y externo.

Palabras clave: dirección estratégica, responsabilidad social empresarial, tiempos emergentes, turismo.

\title{
PROCESS OF STRATEGIC DIRECTION AND CORPORATE SOCIAL RESPONSIBILITY TO ENHANCE TOURISM IN EMERGING TIMES
}

\begin{abstract}
Tourism has been one of the sectors of the economy that has been hit the hardest in times of pandemic, its repercussion has been of a very hostile and direct impact from the economic point of view and of Corporate Social Responsibility towards the workers of the sector and the clients. In this sense, the research aimed at the design of a Strategic Management Process for the development and reactivation of the tourism sector in emerging times with social responsibility, with the aim that organizations are prepared in time to respond to the needs of clients and society based on their economies, finances and social responsibility, since it is highly unlikely that managers and directors can estimate the duration of this type of social phenomenon. For which, the research carried out is descriptive-explanatory with a mixed approach, which allowed transforming data into timely information to make decisions. Likewise, analysis and synthesis methods were used to develop the theoretical and methodological foundation of the study, leaving explicit from this, the strategic proposal for the development of tourism in a specific emergency context, based on a projection that is focused in the management and service to internal and external clients.
\end{abstract}

Keywords: strategic direction, corporate social responsibility, emerging times, tourism.

\section{Proyecto de investigación}

Propiedad Intelectual y Responsabilidad Social Empresarial en la protección de los productos/servicios de las MIPYME's ante el mercado: casos de estudio. 


\section{Introducción}

El turismo es uno de los sectores de la economía que proporciona mayor desarrollo económico a un país, con lo cual, su estudio debe ser integral, para que, en situaciones emergentes ajenos a la voluntad del hombre pueda ser proactivo en las decisiones en cuanto a su situación económica-financiera y de Responsabilidad Social Empresarial (RSE). Esta percepción concuerda con lo expresado por la Organización Mundial del Turismo (OMT, 2010), cuando considera, que "con el paso del tiempo, han surgido y han invertido en el desarrollo turístico un número cada vez mayor de destinos, haciendo del turismo moderno el principal motor del progreso socioeconómico creando empleo y empresas, desarrollando infraestructuras y obteniendo beneficios" (p. 12).

En tal sentido, Ecuador, es un país que trata de potenciar el turismo en todas sus provincias, sin embargo, su proyección no ha sido desarrollada desde un interés del Estado, sino que, ha sido impulsado por capitales privados que han comenzado como emprendimientos y, a través de los años, se han ido consolidando como empresas turísticas desde el punto de vista hotelero y extrahotelero. De esta manera, su funcionamiento ha sido más empírico que basado en la formación y preparación de sus gerentes y empleados, lo cual, ha limitado que su desarrollo sea sostenible en el tiempo y que sus economías y finanzas sean fuertes para soportar impactos directos producto de situaciones emergentes con una responsabilidad social consciente.

Al respecto, se propone como objetivo general diseñar un Modelo Conceptual de Proceso de Dirección Estratégico para el desarrollo y reactivación del sector del turismo en tiempos emergentes con responsabilidad social, con la finalidad, de dar respuesta a las intenciones del Plan de Desarrollo de Turismo Sostenible (PLANDETUR 2020), citado por Villacis (2018), que detalla "el sector turístico ecuatoriano se ha consolidado como uno de los principales generadores de ingresos en Ecuador, junto con el petróleo y el banano y, por lo tanto, merece atención nacional a efectos de mejorar su competitividad y lograr cambios importantes en su estructura" (p. 35).

Con lo cual, se demuestra que existe la necesidad de no solo contar con estrategias de desarrollo en tiempos normales, sino también, considerar propuestas de funcionamiento 
y desarrollo para el sector turístico en situaciones emergentes ajenos a la voluntad del hombre, que den soporte a su sostenibilidad y responsabilidad para sus ciudadanos.

Asimismo, el estudio se justifica a partir de las carencias que presenta el sector turístico en cuanto, a: infraestructura inapropiada para enfrentar situaciones emergentes, limitando compromiso con los trabajadores del turismo a nivel de país y empresarial como parte de la RSE, proyección insuficiente para responder al funcionamiento del turismo para diferentes alternativas de acción, entre otros problemas que no responden al funcionamiento y desarrollo del sector.

En tal sentido, el problema que centra este estudio es ¿Cómo potenciar el turismo en tiempos emergentes? Para ello, el objetivo general se define como Diseñar un Modelo Conceptual de Proceso de Dirección Estratégico para el desarrollo y reactivación del sector del turismo en tiempos emergentes con responsabilidad social, con la finalidad, de que las empresas turísticas, puedan contar con determinadas estrategias que las hagan funcionar en tiempos de normalidad o en situaciones emergentes.

\section{Fundamentación teórica}

Fundamentación de la evolución del Proceso de Dirección Estratégico y su relación con la Responsabilidad Social Empresarial

El Proceso de Dirección Estratégico (PDE) ha ido desarrollándose a través de la historia y, junto con ello, sin que lo hayan explicitado los diversos autores se ha relacionado con el término de Responsabilidad Social Empresarial (RSE). En este sentido, Villacis (2018) remonta su estudio, al pensamiento administrativo que surge cuando las civilizaciones antiguas comenzaban a emplear términos coherentes a la administración, entre los que destacan: planeación, organización, dirección y control, descentralización del poder y organización funcional de poderes administrativos. De esta manera, al considerarse esos aspectos, ya puede percibirse un sentido RSE, pues las cuatro variables mencionadas se enfocan en cualquier organización hacia el sentido de tomar en cuenta no solo los aspectos de la funcionalidad de una empresa, sino también, la de tener presente al ser humano en su atención y consideración. 
En correspondencia a lo anterior, Medina y Ávila (2002) corroboran que, en los años 1800, Watt y Matthew refieren la preocupación de los primeros empresarios por incrementar la productividad, disminuir los costos y considerar el tratamiento del ser humano desde el punto de vista salarial. No obstante, en los años 1900, se toma en consideración con más fuerza la administración científica basada en un enfoque más humanístico del trabajo (denominada enfoque clásico de la administración).

Pero no es hasta los años 1940, que comienza a emplearse términos de dirección estratégica más cercanos a la actualidad, como son: objetivos organizacionales, dirección por objetivos, entre otros, que fortalecen los criterios gerenciales y desarrollan determinados conceptos que conforman lo que hoy en día se denomina proceso estratégico con un sentido más humanista y de RSE, al referirse a los empleados como eje esencial de los resultados empresariales. Asimismo, se coincide con Casate (2007), cuando expone, que, a finales del siglo XX, aumenta el interés por crecer las empresas desde el punto de vista estratégico y competitivo, dado el dinamismo y poderío alcanzado por los países industrializados. Considerándose esta etapa también, de auge para la RSE desde la óptica ambiental y de tratamiento al personal interno de las organizaciones, pues se comienza a emplear el término de talento humano, percibiéndose así, un tratamiento más responsable para el personal de la organización.

En tal sentido, se concuerda con Aguilera (2010) cuando expone que, las empresas están avocadas a enfrentar nuevos desafíos enfocadas a procesos de gestión más eficientes, eficaces y efectivos, en búsqueda de generar beneficios no solo para sí, sino también, para enfrentar los problemas sociales. Es por ello, a partir de la década del 80 el sector turístico toma fuerza y es considerado como la industria sin humo, pues de forma responsable genera ingresos, desarrolla comunidades, ofrece empleos, y contribuye al desarrollo del esparcimiento y a la salud de las personas en su tiempo ocio.

Asimismo, no es hasta el comienzo del siglo XXI, que se incrementa el interés de los empresarios por adoptar un enfoque estratégico más integral, que contribuya al desarrollo de las organizaciones con la participación de todos sus empleados. En este sentido, Martínez y Briceño (2013), consideran que, el enfoque estratégico ingresa como un nuevo modelo de administración, con características que lo fortalecen, entre las que 
destacan: formulación, implementación y evaluación del proceso estratégico, con el propósito de mejorar la gestión y desarrollar la capacidad de los empleados y la empresa en el logro de sus objetivos personales y organizacionales de manera responsable.

Después de las consideraciones anteriores, debe señalarse, que otros autores han realizado aportes importantes dentro del campo del PDE, RSE, Desarrollo Sostenible (ODS, 2015) y el desarrollo del turismo (OMT, 2010), destacándose entre ellos: Frías et al. (2013, 2017); González(2017);Nguyen, (2017);Fonseca (2018); y, García (2018), los cuales, abordaban aspectos relacionados, con: objetivos organizacionales, desarrollo del talento humano, responsabilidad social organizacional, bienestar social, inversión social responsable, entre otros aspectos que caracterizan el grado de integración que tienen que tener los procesos estratégicos para cualquier sector de la economía.

En consecuencia, el enfoque estratégico del sector y las empresas turísticas deben proyectarse en función de la RSE y la sostenibilidad de las organizaciones, con la finalidad, de que contribuyan al bienestar de sus empleados y la sociedad con una visión interna y externa de la misma, definiéndose su proyección para cualquier situación o contexto, ya sea, para tiempos normales y emergentes en favor del entorno social. Razón por la cual, hay períodos de tiempo de funcionalidad que son fundamentales para la definición e implementación de estrategias enfocadas en la competitividad, cambios tecnológicos, atención al personal interno y externos (clientes), responsabilidad económica de las organizaciones y sus empleados, sostenibilidad de las empresas en el tiempo, entre otros factores a considerar en la propuesta, donde no se pierda el nivel de competitividad ni de imagen de estas ante el mercado.

Asimismo, es importante los criterios de Navas y Guerras (2012) con respecto a diferentes tipo de niveles de estrategias, entre las que destacan: a) Estrategia competitiva o negocio, que determina cómo competir mejor en los distintos negocios; b) Estrategia a nivel corporativo o empresa, direcciona el futuro de la empresa; c) Estrategia funcional, se centran en cómo utilizar y aplicar los recursos y habilidades dentro de cada área funcional, con el fin, de maximizar la productividad de dichos recursos. A lo planteado, debe incluirse las estrategias para situaciones emergentes con RSE, que deben estar encaminada a enfrentar los problemas más complejos, respecto a: empleados, clientes, sostenibilidad económica en el tiempo, entre otros aspectos a considerar. 


\section{Metodología}

El estudio contempla una investigación de tipo descriptiva-explicativa, empleándose para ello, métodos teóricos y prácticos, entre los que sobresalen: análisis-síntesis, inductivo-deductivo, histórico-lógico, análisis documental, entre otros, que confirman la teoría abordada por otros autores Frías et al. (2017) y Villacis (2018) y la necesidad de proponer nuevas estrategias para el sector del turismo en situaciones emergentes las cuales fueron confirmadas mediante la información obtenida en una encuesta aplicada a los diferentes cantones de una provincia objeto de estudio en el Ecuador.

Asimismo, se logra analizar la forma en que debe ejecutarse la estrategia a nivel provincial, y trazar los objetivos, así como las acciones que potencien el turismo en cada uno de los cantones objeto de estudio en el país, evidenciándose la necesidad aplicar estrategias que enfrenten situaciones complejas desde el punto de vista de la satisfacción de las necesidades, gustos y preferencias de los turistas y visitantes en contextos emergentes, a partir de la sostenibilidad económica que deben soportar, pero también mantener las organizaciones para que no decaiga, este importante renglón de la economía del país y la provincia.

En tal sentido, la encuesta aplicada a directivos de los nueve cantones se relaciona con un enfoque mixto, dada la finalidad, de hacer un análisis cuantitativo y cualitativo que se oriente a observar el comportamiento de diversas variables relacionadas al tema objeto de estudio en función del proceso estratégico y de la RSE para el sector turístico, donde algunos de los resultados del análisis de las encuestas, se muestran en el siguiente apartado.

\section{Resultados}

Existe un $80.18 \%$ de coincidencia entre los implicados en el estudio, que determinan, que las empresas turísticas no estaban preparadas económica y financieramente para enfrentar la sostenibilidad de las organizaciones por períodos prolongados en situaciones emergentes (por ejemplo, caso pandemia COVID-19). 
E1 93.3\% de los implicados, confirman la necesidad de que los gerentes en situaciones de funcionamiento normal del país deben crear fondos de reservas sólidos para enfrentar situaciones emergentes con responsabilidad, con la finalidad, de evitar despidos masivos de empleados en el sector.

A su vez, el $87.6 \%$ de las personas, consideran que, la formación de gerentes y personal administrativo es insuficiente para determinar proyecciones y enfrentar diversos contextos de la vida política y económica de un país, en cuanto a la repercusión de sus negocios a partir de los impactos internos y externos que ocurren producto de la globalización, incluyéndose los fenómenos naturales.

El $96.25 \%$ de los encuestados coinciden en que, no existe una proyección estratégica de mediano y largo plazo, que contribuya a la sostenibilidad económica y financiera de la organización, lo cual, trae consigo que las primeras medidas que se tomen en situaciones complejas sean reducción de plantillas y cierres o quiebres de empresas.

Asimismo, el $84.75 \%$ de las personas exponen que, no existen en el sector turístico, específicamente en las empresas, procesos de dirección estratégicos que garanticen calidad, responsabilidad y competitividad, dada, la formación empírica de sus emprendedores, lo cual, afecta directamente la falta de responsabilidad de los empleadores con sus empleados y clientes, que demandan satisfacer necesidades tanto desde el punto de vista de trabajo como de recreación en estos últimos.

El $87.35 \%$ de los encuestados, opinan que, en situaciones emergentes prolongadas, se quiere reactivar el sector turístico sin un total sentido de la responsabilidad, pues no tienen en cuenta sus promotores el riesgo de los clientes internos y externos, el grado de afectación de las economías de los clientes externos al momento de pagar un servicio turístico, la baja calidad del servicio que se recibe a partir del despido de empleados por temas financieros de no poder pagar todos los salarios, entre otros aspectos que influyen directamente en las personas.

A su vez, el $100 \%$ de los encuestados, detallan que, no existe ayuda, colaboración ni proyección del Estado para lograr una reactivación económica del sector turístico, con 
lo cual, cada empresario debe responder a sus emergencias y necesidades de funcionar. Esto ha conllevado, a que diversas empresas turísticas hayan desaparecido en situaciones emergentes prolongadas.

En tal sentido, y dado los análisis y resultados obtenidos en el estudio, se propone un proceso de dirección estratégico con responsabilidad social para reactivar el turismo en la provincia de Tungurahua, donde, la propuesta del proceso de dirección estratégico se fundamenta en determinados principios, objetivos, características y condiciones básicas para su aplicación en las empresas del sector turístico, los cuales se describen a continuación:

\section{Principios del Proceso de Dirección Estratégico:}

- Carácter interactivo basado en la integración de los procesos y actividades en las empresas turísticas que garanticen un nivel de atención adecuado a los clientes internos y externos en cualesquier circunstancia con responsabilidad social.

- Formación y actualización personal especializado en la empresa, para que pueda aportar y trabajar con responsabilidad social durante todo el proceso de formación-acción en la prestación de sus servicios.

- Mantener niveles de atención personalizada en cualquier situación de funcionalidad, con la finalidad, de ofrecer un servicio de calidad y responsable.

- Funcionalidad del proceso de dirección estratégico para la gestión y la toma de decisiones responsable con carácter proactivo y en beneficio de todos los actores (empresarios, empleados, clientes y sociedad).

\section{Objetivos del Proceso de Dirección Estratégico:}

- Evaluar la situación del país y el contexto internacional para la gestión y el análisis de los resultados de las estrategias para su futura proyección. 
- Desarrollar la formación del personal del sector turístico para enfrentar situaciones de funcionalidad en momentos de normalidad y emergencias para el corto, mediano y largo plazo, lográndose con ello, que los directivos y empleados del turismo, reconozcan la utilidad del proceso de dirección estratégico con responsabilidad, potenciándose así, la planeación y ejecución de las actividades turísticas en todos los cantones.

- Incrementar los niveles de gestión estratégica turísticas para el desarrollo económico-social de la provincia en situaciones de normalidad y emergencia.

- Desarrollar procesos de retroalimentación efectivos para la toma de decisiones proactivas y efectivas en todas las empresas turísticas, con la finalidad, de emplear de manera eficiente la información que genera el proceso de dirección estratégico.

\section{Características del Proceso de Dirección Estratégico:}

Pertinencia: contextualizado a la operacionalización de los problemas que pueden presentarse en las empresas turísticas en un período determinado.

Flexibilidad: aplicado a todas las empresas que trabajen en el sector con sentido estratégico y de responsabilidad social a corto, mediano y largo plazo.

Calidad: debe contribuir al beneficio de la sociedad y de todos los empleados de la organización en cualesquiera de las circunstancias, sobre todo, en tiempos emergentes.

Consistencia: priorizar los procesos que generan responsabilidad social en situaciones de emergencias de una forma lógica sin afectar la calidad del servicio de la red turística.

Contextualizado: flexible a la situación que vive el país en tiempos emergentes.

Integrador: relacionarse con otros sistemas de gestión, de forma tal, que contribuya al mejoramiento continuo de los procesos que se ejecutan. 


\section{Condiciones básicas para el diseño e implementación del Proceso de Dirección Estratégico:}

1. Se diseña y rediseña de acuerdo con la situación que tiene cada instalación turística y debe ser integrador a otros sistemas de gestión para la toma de decisiones proactivas.

2. Ser eficaz, sea, debe concentrar su atención en los procesos y actividades que generan valor para el cliente (turistas y visitantes), y de fácil comprensión para los usuarios (directivos y empresarios).

3. Los beneficios que genera deben encaminarse al desarrollo del turismo.

A partir de lo expuesto, se propone el Proceso de Dirección Estratégico a partir de un modelo conceptual para potenciar el turismo en situaciones emergentes (ver esquema 1), el cual, responde a la contextualización del desarrollo estratégico que debe ejecutar el Comité Provincial de Turismo en la provincia de Tungurahua como encargados contribuir al progreso social de los territorios.

El proceso de dirección estratégico planteado en el modelo conceptual, considera un proceso de retroalimentación proactivo para tomar decisiones oportunas en el menor tiempo posible en situaciones emergentes con responsabilidad social, con la finalidad, que ni la organización ni sus integrantes acusen de problemas e imprevistos a última hora dada la prolongación que pueda tener el fenómeno evento presentado.

En su primer nivel, destaca el diagnóstico interno y externo de la organización para estar orientados sobre la situación del turismo en la provincia y los cantones en una situación emergente determinada. Este nivel es considerado como estratégico, pues permite visualizar y proyectar el desarrollo del turismo desde una perspectiva interna y externa que llegue a todas las instituciones y empresas turísticas.

En tal sentido, el proceso estratégico concebido, parte de la misión y visión de las empresas turísticas, donde se destacan algunos aspectos que deben considerar con total responsabilidad social, pues implica: sostenibilidad del negocio aunque la situación emergente sea prolongada; cuidado de los empleados para que conserven sus puestos 


\section{Esquema 1}

Proceso de Dirección Estratégico a partir de un modelo conceptual para potenciar el Turismo

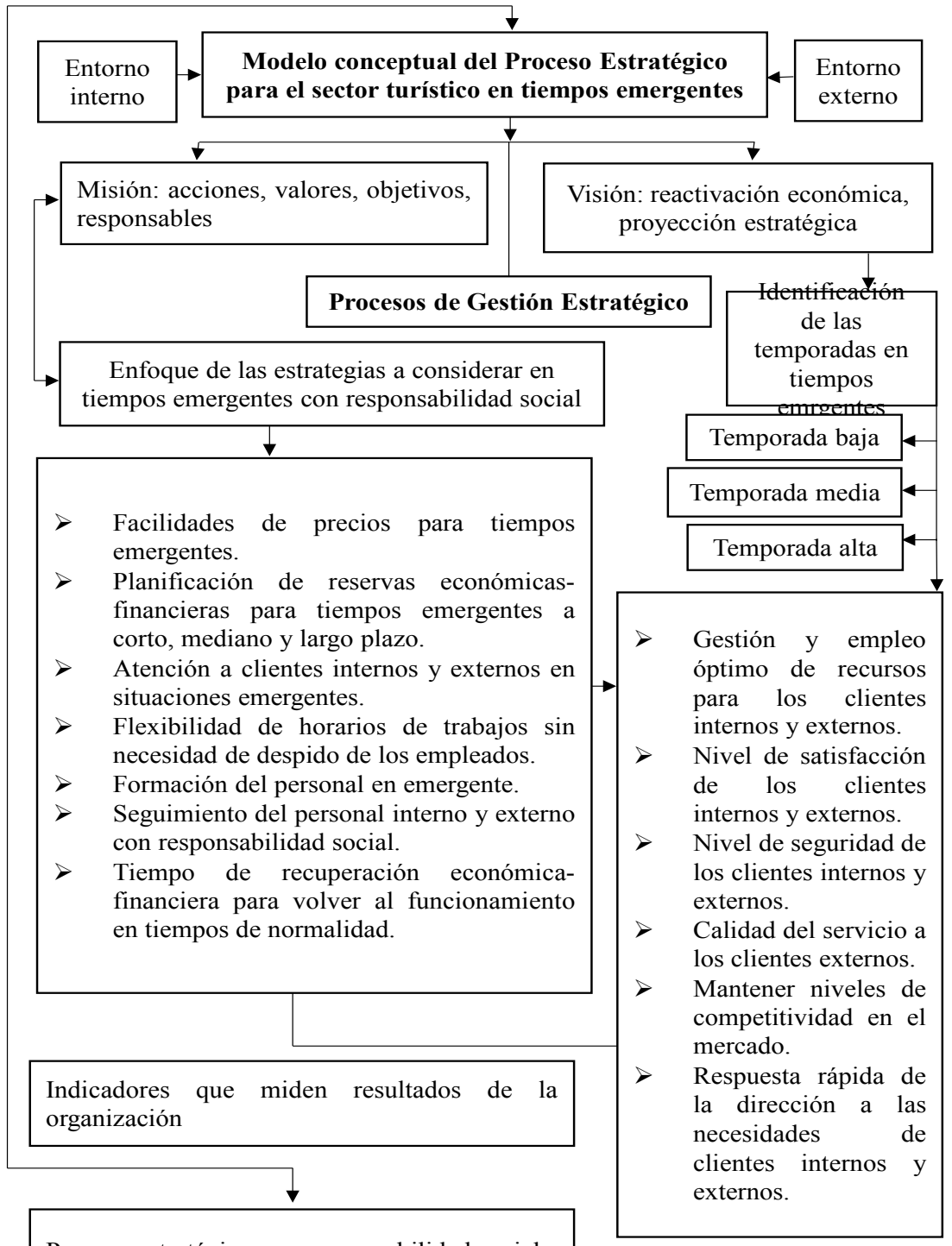

Fuente: elaboración propia (2020). 
de trabajo; satisfacción de los clientes externos que estén presenten en las instalaciones durante la situación emergente, y conservación de una planeación objetiva y alcanzable en cuanto a comportamientos de indicadores razonables para situaciones de emergencias.

En la ejecución del proceso estratégico, deben estar correctamente interconectadas las acciones, valores, objetivos e indicadores alcanzables por la organización, a partir de una planificación adecuada de todos los recursos que deben estar disponibles en situación de emergencia para prestar un servicio de calidad.

El Proceso de Dirección Estratégico concebido puede ser adoptado jerárquicamente desde el nivel de provincia hasta el nivel organizacional en función de las características propias de cada cual, asimilándose así, la atención que deben recibir los clientes internos y externos que demandan determinados servicios. Esto trae consigo, que se promueva desde la concepción y adopción del modelo una diferenciación de actividades a ejecutar y una atención personalizadas con todos los implicados para dar respuesta proactiva a los problemas sociales que puedan acontecer.

Asimismo, el nivel de operacionalización de los procesos a considerar debe enmarcarse en el cumplimiento de indicadores razonables en cuanto a su proyección se refiere, o sea, estos deben ser alcanzables objetivamente dado la situación de emergencia que se vive por parte de la organización. Esto trae consigo, que las empresas turísticas deben trabajar sobre todo con mayor ahínco en convertir las temporadas medias o bajas del turismo en temporadas asequibles para los turistas o clientes externos, proyectándose en sus propuestas un carácter innovador y tecnológico, donde despierte el interés de las personas por consumir sus productos y/o servicios. Por ejemplo, desde el punto de vista económico: otorgar mayor amplitud de facilidades de pagos a los clientes o turistas, mejorar los precios, mantener las reservaciones realizadas con anterioridad a la situación de emergencias, ofrecer seguridad de recibir un servicio rápido o una respuesta adecuada en forma personalizada, entre otros aspectos.

Al respecto, se dejan planteados algunos objetivos estratégicos que pudiesen contribuir al mejoramiento de la reactivación del turismo en situaciones emergentes con responsabilidad social en la provincia de Tungurahua, Ecuador: 


\section{a) Objetivos de la provincia de Tungurahua}

- Asociar instituciones y empresas turísticas para mantener el servicio turístico en situaciones emergentes.

- $\quad$ Atender de manera personalizada a los turistas y visitantes que se encuentran recibiendo servicios durante la situación emergente con responsabilidad social, proveyéndoles de todos los recursos que necesitan en ese momento que se vive.

- Promover servicios turísticos diferenciados en tiempos emergentes no descuidándose la vida de los clientes.

- Incrementar el nivel de información adecuado a los clientes sobre la situación emergente que se vive diariamente.

- Formar a los profesionales para situaciones emergentes, con la responsabilidad de que puedan dar respuesta proactiva a sus clientes.

- Desarrollar políticas de seguridad y gestión de riesgos para las empresas turísticas, empleados del turismo, comunidad turística y turistas en sentido general.

- $\quad$ Fortalecer el mantenimiento de las instalaciones turísticas durante el período que dura la situación emergente.

- Alinear la gobernabilidad en función del proceso estratégico, incrementándose con ello, la transparencia de las informaciones relacionadas a: protección de los clientes internos y externos, conservación del medio ambiente, mantenimientos y limpieza de las instalaciones, entre otros aspectos.

\section{Los objetivos planteados, pueden alcanzarse considerándose lo siguiente:}

- Planificación del turismo en tiempos emergentes. 
- Seguridad y cuidado de los clientes internos y externos en situaciones emergentes.

- Diferenciación de productos y servicios para situaciones emergentes.

- Movilidad segura según las condiciones de la situación emergente.

- Formación y apoyo a la mejora empresarial desde los organismos centrales del Estado.

- Regulaciones de funcionamiento contextualizadas para el funcionamiento en tiempos emergentes.

- Mantenimiento del bienestar social de todos los empleados que laboran en el sector turístico.

Una vez considerado los objetivos y algunas estrategias que se pueden contemplar en situaciones emergentes con responsabilidad social proponen comparar los resultados con algunas percepciones tratadas en la literatura consultada.

\section{Discusión}

Los aspectos tratados en la investigación corroboran que, el tema es pertinente y que es necesario para que todas las organizaciones tengan en cuenta su proyección estratégica con responsabilidad social no solamente en tiempos de normalidad, sino también, en situaciones emergentes como la que se vive en la actualidad con la Pandemia COVID-19, que ya es considerada en el tiempo para la sostenibilidad económica de las mismas.

En tal sentido, se realiza un análisis a partir del estudio de un conjunto de propuestas de proyección estratégica relacionadas al sector del turismo por algunos autores reconocidos, entre los que se destacan: Frías et al. (2013, 2017); González (2017); y García (2018). Estos autores, consideran una serie de factores que son necesarios contemplar dentro de todo proceso de proyección estratégica con responsabilidad social, con la finalidad, de mejorar el grado de efectividad que se puede lograr en cada empresa 
turística con la participación de todos los miembros de la organización, pensándose siempre en la responsabilidad del sector con respecto al aporte y al desarrollo económico financiero que se debe alcanzar en el territorio. Percepciones, con la cual, coinciden los autores de esta investigación, respecto a:

- La proyección estratégica de las empresas dedicadas a la actividad turísticas concibe el desarrollo de la comunidad y el territorio donde se desenvuelven con responsabilidad social, pues constituye una fuente de empleo para sus pobladores.

- El proceso estratégico en tiempos emergentes debe responder a la seguridad y protección de los clientes internos, con la finalidad, de que los procesos internos no se afecten durante la situación excepcional y se pueda atender a los clientes externos, sin que perciban niveles de preocupación o estrés mientras reciban el servicio demandado.

- El proceso estratégico debe estar enfocado a la búsqueda de la estabilidad organizacional, a la conversión de lo intangible en tangible en la prestación del servicio, a estimular el empoderamiento de los clientes internos en la realización de sus funciones, a la generación de valor cuantitativo y cualitativo para la organización y los clientes externos.

- Todos los procesos estratégicos con nivel de responsabilidad se enfocan en la administración eficiente, eficaz y efectivo para la mejora continua y el logro de sus objetivos consensuados a partir de la ejecución de sus procesos y actividades.

Sin embargo, algunos estudios en la literatura consultada y mencionada en la fundamentación teórica (D’Alessio (2008); Navas y Guerras, 2012; Martínez y Briceño (2013); Bolaños et al. (2014); Nguyen, (2017); Fonseca (2018); Villacis (2018); hacen referencia a la preocupación netamente de los empresarios por obtener solo resultados económicos-financieros, sin reparar en aspectos de responsabilidad social como, por ejemplo: incremento de la productividad, reducción de los costos, reajuste de plantilla, disminución de servicios, entre otros. 
En tal sentido, los autores del estudio no concuerdan, porque el proceso estratégico de las empresas turísticas no debe dejar de pensar responsablemente desde su concepción en el ser humano, ya sea, en lo clientes internos como en los externos en tiempos emergentes.

Asimismo, los procesos estratégicos deben contemplar desde un estado de normalidad funcional del sector turístico la posibilidad de proyectar responsablemente la sostenibilidad de las organizaciones en el tiempo, tratándose de evitar el quiebre de las mismas por el factor económico relacionados a los ingresos y la rentabilidad.

\section{Conclusiones}

El proceso de dirección estratégico se concibe con el criterio de todos los implicados en el sector turístico, dado que, son estos los comprometidos con la sostenibilidad en el tiempo de las empresas y su funcionalidad en tiempos emergentes de manera responsable. Lo cual contribuye a que el modelo propuesto funcione y sea sostenible en tiempo de normalidad y de emergencia, siempre pensándose en el beneficio social de los empresarios, los clientes y la comunidad.

El modelo conceptual del proceso estratégico concibe tres niveles esenciales que garantizan su funcionalidad desde el punto de vista estratégico, táctico y operativo con un proceso de retroalimentación que integra el contexto interno y externo asociados a la organización, concediéndole así, un carácter sistémico. Con lo cual, se completa de manera integral para su desarrollo y toma de decisiones en cada área de la organización, lográndose con ello, que los resultados reflejen beneficios económicos globales para las empresas turísticas y para sus beneficiarios.

\section{Referencias bibliográficas}

Aguilera, A. (2010). Direccionamiento estratégico y crecimiento empresarial: algunas reflexiones en torno a su relación*. Pensamiento \& Gestión (28), 85-106.

Bolaño, Y., Robaina, D., Pérez, A., y Arias, M. (2014).Modelo de Dirección Estratégica basado en la Administración de Riesgos. Ingeniería Industrial, 344-357. 
Casate, R. (2007). La dirección estratégica en la sociedad del conocimiento. Parte I. El cuadro de mando integral como herramienta para la gestión. Acimed (6), 15.

D’Alessio, F. (2008). El proceso estratégico. Un enfoque de gerencia. Pearson Education.

Fonseca, J. (2018). La gestión territorial para el desarrollo sostenible. Caso Cantón Baños de Agua Santa, Tungurahua, Ecuador. Universidad de Matanzas, Cuba.

Frías, R., González, M. y González, M. (2013). Metodología de validación de un Modelo de Gestión del Cliente Interno en procesos empresariales. Res Non Verba, Vol. 4, No.4, ISSN: 1390-6968, ECOTEC.

Frías, R., González, M., Almenares, R. y Ruiz, J. (2017). Gestión de riesgo y alineamiento estratégico en procesos hoteleros seleccionados. Revista Retos Turísticos. vol. 16 (1). enero-abril, 23-38 pp. RNPS: 2367, ISSN: 2224-7947.www. retos.umcc.cu.

García, Y. (2018). Contribución a la gestión de la inocuidad de los alimentos en servicios gastronómicos, periodo de 1997-2018. Editora LOGICUBA "La Habana". ISBN: 978-959-7191-62-9.

González, M. (2017). Modelo de gestión del cliente interno en procesos hoteleros desde el endomarketing, periodo de 1997-2017. Editora LOGICUBA "La Habana”. ISBN: 978-959-7191-62-9.

Martínez, E. y Briceño, M. (2013). Estado del arte del direccionamiento estratégico. SIGNOS, 5(1), 91-102.

Medina, A. y Ávila, A. (2002). Evolución de la teoría administrativa. Una visión desde la psicología organizacional. Revista Cubana de Psicología, 19(3), 262-272.

Organización Mundial del Turismo. (2010). Panorama OMT del turismo internacional. p. 12. 
Navas, J. y Guerras, L. (2012). Fundamentos de dirección estratégica de la empresa. España: S. T. Civitas Thomson.

Nguyen, Dinh Loc. (2017). The Application of Talent Management Theories to the Prevention of "Brain Drain" in China. (Bachelor of Business Administration), Helsinki Metropolia University of Applied Sciences, Metropolia Business School (1406653).

Objetivos de Desarrollo Sostenible (ODS, 2015). Programa de Las Naciones Unidas.

Villacís, M. (2018). Proceso de Dirección Estratégico para potenciar el turismo en la provincia de Tungurahua. Proyecto de investigación previo a la obtención del título de Ingeniera Comercial. Dirigido por Dr.C. Osmany Pérez Barral. Pontificia Universidad Católica del Ecuador Sede Ambato, Ecuador. 